\title{
XMM-Newton observations of the BL Lac MS 0205.7+3509: A dense, low-metallicity absorber
}

\author{
D. Watson ${ }^{1,2}$, B. McBreen ${ }^{3}$, L. Hanlon ${ }^{3}$, J. N. Reeves ${ }^{4,5}$, N. Smith ${ }^{6}$, E. Perlman ${ }^{7,8}$, J. Stocke ${ }^{9}$, and T. A. Rector ${ }^{10,11}$ \\ 1 Niels Bohr Institute, Astronomical Observatory, University of Copenhagen, Juliane-Maries Vej 30, 2100 Copenhagen $\varnothing$, \\ Denmark \\ 2 X-Ray Astronomy Group, Department of Physics and Astronomy, Leicester University, Leicester LE1 7RH, UK \\ 3 Dept. of Experimental Physics, University College Dublin, Dublin 4, Ireland \\ ${ }^{4}$ Laboratory for High Energy Astrophysics, Code 662, NASA Goddard Space Flight Center, Greenbelt, MD 20771, USA \\ 5 Universities Space Research Association \\ ${ }^{6}$ Dept. of Applied Physics and Instrumentation, Cork Institute of Technology, Cork, Ireland \\ 7 Joint Center for Astrophysics, University of Maryland, Baltimore County, 1000 Hilltop Circle, Baltimore, MD 21250, USA \\ 8 Johns Hopkins University, Baltimore, MD 21218, USA \\ 9 Center for Astrophysics and Space Astronomy, University of Colorado, Boulder, CO 80309-0389, USA \\ ${ }^{10}$ National Optical Astronomy Observatory, 950 N. Cherry Ave., Tucson, AZ 85719, USA \\ 11 University of Alaska, 3211 Providence Dr., BMB 212, Anchorage, AK 99508, USA
}

Received 20 June 2003 / Accepted 26 January 2004

\begin{abstract}
The high-frequency-peaked BL Lac, MS 0205.7+3509 was observed twice with XMM-Newton. Both X-ray spectra are synchrotron-dominated, with mean $0.2-10 \mathrm{keV}$ fluxes of $2.80 \pm 0.01$ and $3.34 \pm 0.02 \times 10^{-12} \mathrm{erg} \mathrm{cm}^{-2} \mathrm{~s}^{-1}$. The X-ray spectra are well fit by a power-law with absorption above the Galactic value, however no absorption edges are detected, implying a low metallicity absorber $\left(Z_{\odot}=0.04_{-0.01}^{+0.03}\right)$ or an absorber with redshift above one (best-fit $z=2.1$ for an absorber with solar abundances). In either case the absorbing column density must be $\sim 9 \times 10^{21} \mathrm{~cm}^{-2}$. A new optical spectrum is presented, with a Mg II absorption doublet detected at $z=0.351$, but no other significant features. The optical spectrum shows little reddening, implying a low dust to gas ratio in the absorber. MS 0205.7+3509 must therefore be viewed through a high column density, low-metallicity gas cloud, probably at $z=0.351$ and associated with the galaxy that has been shown to be within $\sim 2$ " of the BL Lac.
\end{abstract}

Key words. galaxies: BL Lacertae objects: individual: MS 0205.7+3509 - galaxies: active - X-rays: galaxies

\section{Introduction}

Blazars are divided into BL Lacs and quasars (either flatspectrum radio-loud, optically violently variable, highlypolarised or core-dominated) based on the strength of emission lines in the optical spectrum (Scarpa \& Falomo 1997, and references therein).

It has been suggested that sources with BL Lac characteristics are actually gravitationally microlensed quasars (Nottale 1986; Ostriker \& Vietri 1990). In these cases, it is expected that stellar mass lenses in a foreground galaxy significantly amplifies the central QSO continuum source but not the emission from the line-emitting regions and that variations in the relative source-lens position could account for the rapid variability observed in many BL Lacs. Sources of this kind should clearly have foreground galaxies, which would result in an apparent decentering of the AGN from the "host" and an

Send offprint requests to: D. Watson, e-mail: darach@astro.ku.dk excess of absorption in these sources. However, the suggestion that BL Lacs are gravitationally microlensed quasars can be discounted for most BL Lacs (Padovani 1992) and only a few remain as possible or probable candidates, most notably AO 0235+164 which appears to have foreground absorption (Abraham et al. 1993; Madejski 1994; Madejski et al. 1996) and a companion AGN (Burbidge et al. 1996). Other candidates include PKS 0537-441 (which shows rapid microvariability, but does not show evidence for a foreground object in optical imaging or spectroscopy, Romero et al. 1999; Pian et al. 2002), and B2 1308+326 which has characteristics intermediate between BL Lacs and quasars (Gabuzda et al. 1993; Watson et al. 2000), but where high resolution imaging of the BL Lac with the HST WFPC2 (Urry et al. 1999) was consistent with a point source.

MS 0205.7+3509 is another such rare candidate, and while deep imaging has revealed that the BL Lac is centred on a host galaxy that is likely an elliptical and not offset in a spiral host 
as had been inferred from previous observations (Stocke et al. 1995), a companion galaxy was also detected very close to the BL Lac line of sight (Falomo et al. 1997) which had caused the previous inference of decentering in a spiral host to be made. X-ray observations with ROSAT (Stocke et al. 1995) and ASCA (Watson et al. 1999) showed the existence of absorption well above the Galactic level, indeed at a level second only among BL Lacs to PKS 1413+135 (Perlman et al. 2002). These results, on MS 0205.7+3509, imply that the X-ray absorber is in the companion galaxy which is foreground to the AGN. It has been suggested that stars in the halo of this companion galaxy could be responsible for microlensing of the BL Lac (Falomo et al. 1997; Watson et al. 1999).

In spite of the relatively good spectral resolution of $A S C A$, the redshift of the absorber could not be constrained from those observations (Watson et al. 1999). A redshift of $z=0.318$ was proposed based on the tentative detection of a Ca II absorption system reported in the optical spectrum of this source (Morris et al. 1991) indicating the possible redshift either of the host galaxy or of a foreground absorber and to date this redshift had been used as the best available (Rector et al. 2000; Stocke et al. 1995).

Though somewhat lower in terms of column density, MS 0205.7+3509 is six times brighter in X-rays than PKS 1413+135, making it one of the best available cases in which to study absorption in the hot phase of the ISM in a galaxy that is not at low redshift. XMM-Newton observations were performed in an attempt to determine the nature of the X-ray absorber in MS $0205.7+3509$, in particular in the context of a foreground lensing galaxy.

Results from these data are presented in this paper. Section 2 deals with the observations and the data reduction procedures; results from the X-ray and optical data are in Sect. 3. A discussion of these results and a summary of our conclusions are given in Sect. 4. Uncertainties given are $90 \%$ confidence limits unless otherwise stated. A flat universe with $H_{0}=75 \mathrm{~km} \mathrm{~s}^{-1} \mathrm{Mpc}^{-1}$ and $\Omega_{\Lambda}=0.7$ are assumed throughout.

\section{Observations and data reduction}

MS 0205.7+3509 was observed by XMM-Newton (Jansen et al. 2001) for $40 \mathrm{ks}$ and $20 \mathrm{ks}$ during orbits 217 and 395 with observations starting on 14 Feb. 2001, 07:06:41 UT and 4 Feb. 2002, 19:41:06 UT respectively.

For the first observation, exposures of $38 \mathrm{ks}$ and $34 \mathrm{ks}$ duration were made with each EPIC-MOS (Turner et al. 2001) and with the EPIC-pn (Strüder et al. 2001) detector respectively; while for the second observation, the exposures were $17 \mathrm{ks}$ (each EPIC-MOS) and $15 \mathrm{ks}$ (EPIC-pn). All observations were performed in Full Frame mode, using the Medium filter for the MOS cameras and the Thin filter for the pn. Data were processed and screened in a standard way with the XMM-Newton Science Analysis System version 5.3.0 (SAS). Only events corresponding to patterns 0-12 were used for the two MOS cameras, while pattern 0-4 events were selected from the pn data.

A circular extraction region was defined around the centroid position of the source, with an aperture of $35^{\prime \prime}$ radius. Data from these spatial regions were used to extract spectra and lightcurves. An estimate of the background was derived by using an aperture of 100" radius at a source-free position close to the source extraction region.

The complete lightcurves were created by binning the data in $70 \mathrm{~s}$ bins (giving $\sim 150$ counts per bin); two other, different time binnings yielded similar results. The background for each instrument was subtracted from the source lightcurve and the per-instrument lightcurves were added.

The extracted spectra were binned to give a minimum of twenty counts per bin. Data in the energy range $0.2-10.0 \mathrm{keV}$ were used. The background-subtracted spectrum from each instrument was fit separately using the Levenberg-Marquardt algorithm in Xspec v11.0.1 and EPIC response matrices generated with the SAS.

The EPIC-pn $0.2-10.0 \mathrm{keV}$ count rate was found to be between $1.2-1.5$ counts/s; effects due to photon pile-up are negligible at this flux level ( $\$ 0.2 \%$ of the total counts).

Differences between the spectra extracted from the EPIC-MOS cameras are negligible in this observation. The MOS data were therefore co-added and fit as a single spectrum, using the co-added response matrices and backgrounds. However, known differences in the cross-calibration of the EPIC-pn and MOS instruments are apparent in these spectra (Fig. 2), these differences primarily affect the low-energy power-law slopes and normalisations. In order to exploit the full dataset, the MOS and pn spectra have been fit separately and then simultaneously and any significant differences have been highlighted.

\subsection{Optical}

An optical spectrum (Fig. 2) was obtained in a 9200 s exposure completed on 23 November 2000 with the Blue Channel spectrograph at the $\mathrm{MMTO}^{1}$. The slit width was $1^{\prime \prime} .25$, resulting in a FWHM of $4.2 \AA$. The slit position was E-W. The signal to noise ratio is $\sim 20$. The spectrum was extracted using the IRAF kpnoslit package. The spectra were wavelength calibrated with HeNeAr spectra, with a rms in the solution of about $0.25 \AA$. The spectra were flux calibrated with observations of the standard stars Feige 34 and G191B2B.

No emission features were identified ( $W_{\lambda} \lesssim 3 \AA$ ) in the spectrum. However, an absorption doublet, identified as $\mathrm{Mg}$ II at $z=0.351$, is detected. The first line of the doublet is at $3776.8 \AA$, with an equivalent width $\left(W_{\lambda}\right)$ of $1.3 \pm 0.1 \AA$. The second line is at $3786.4 \AA, W_{\lambda}=1.2 \pm 0.1 \AA$. The doublet spacing is correct for the $\mathrm{Mg}$ II doublet at $z=0.351$ (Fig. 2 inset). The individual line widths are not resolved however. A second, low significance absorption system at $3510 \AA$, corresponding to Fe II $2600.17 \AA$ at $z=0.351$ is also marginally detected $\left(W_{\lambda}=1.6 \pm 0.2 \AA\right)$. We note that the previously reported redshift $(z=0.318)$ was tentative and the spectrum very featureless, with lower signal-to-noise ratio than the spectrum presented here (Morris et al. 1991).

1 The MMT Observatory is a joint facility of the University of Arizona and the Smithsonian Institution. 


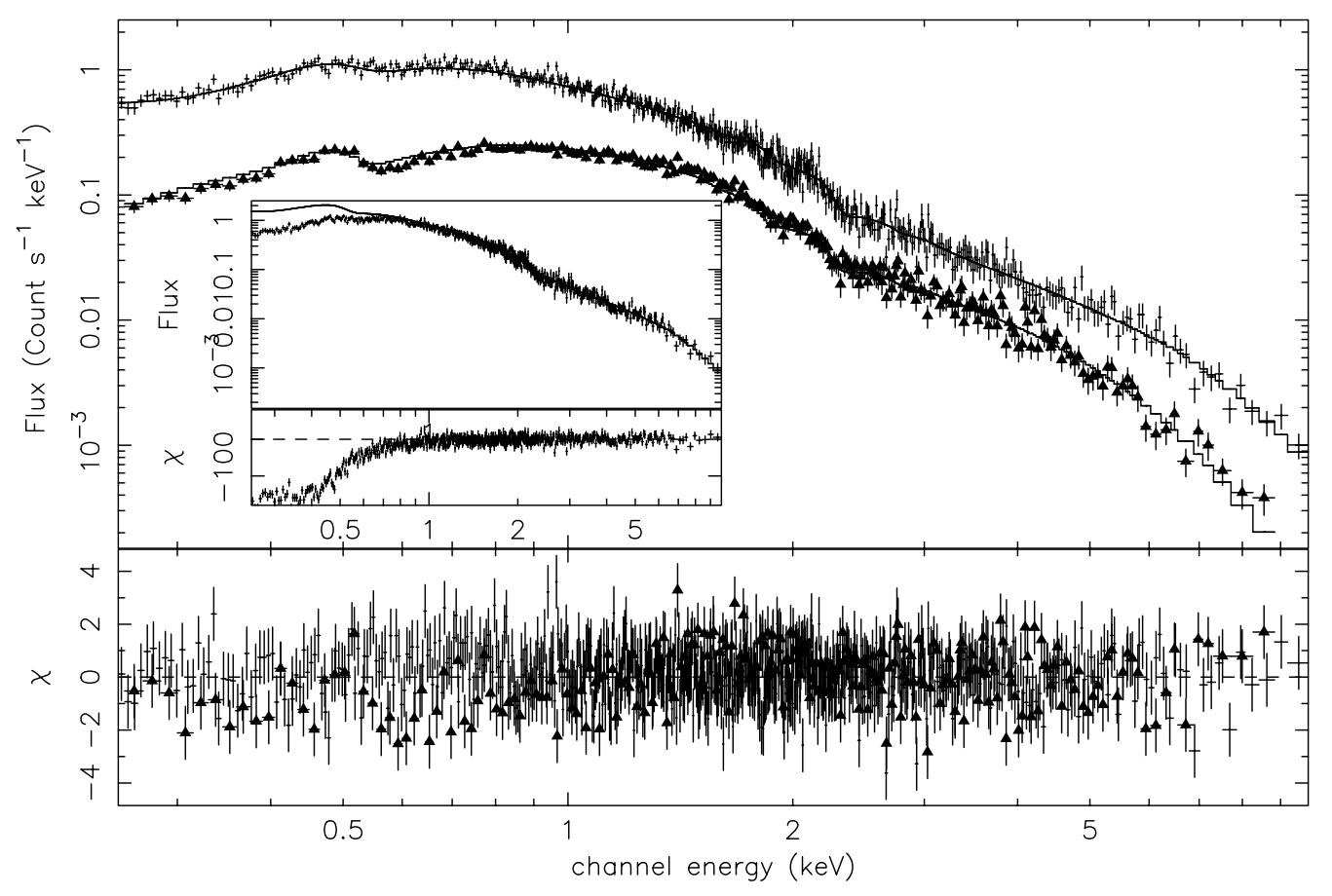

Fig. 1. Simultaneous fit of EPIC-pn (crosses) and combined EPIC-MOS (triangles) data from 14 Feb. 2001 folded through the detector response with fit residuals. The model fit was a Galacticabsorbed power-law with neutral low-abundance absorption at redshift $z=0.351$. The elemental abundance and absorbing column were fit simultaneously in both datasets (from 14 Feb. 2001 and 4 Feb. 2002), whereas the power-law slopes and normalisations were allowed to vary independently to account for the temporal variability of the synchrotron spectrum. Inset. Power-law model fit to the $1-10 \mathrm{keV}$ EPIC-pn data of 14 Feb. 2001 with Galactic absorption only.

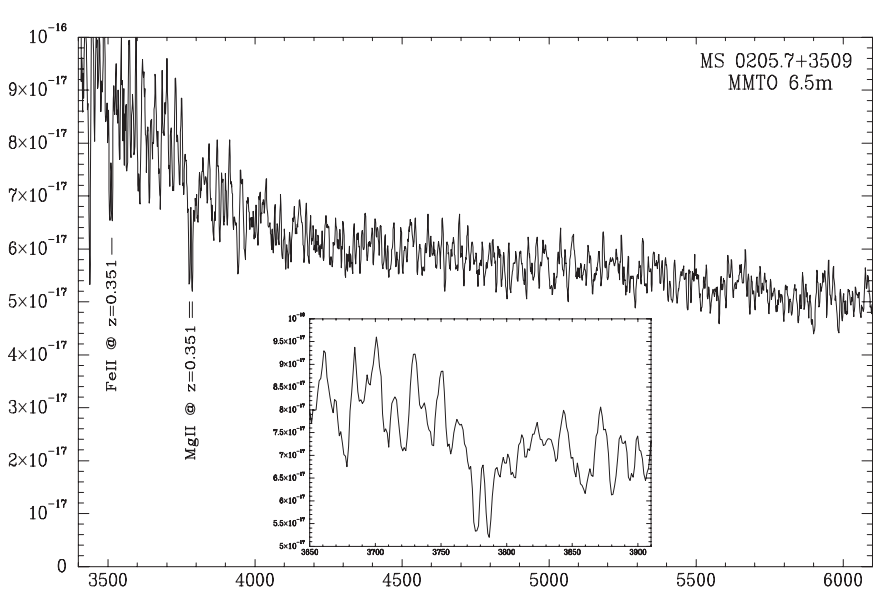

Fig. 2. Optical spectrum from the MMTO. Absorption lines of Fe II and $\mathrm{Mg}$ II at $z=0.351$ are labelled. Inset. A blow-up centred on the resolved Mg II doublet. Wavelength $(\AA)$ is plotted on the axis of abscissas, flux (arbitrary units) on the ordinate. (The deviations from a power-law shape at the blue and red ends of the spectrum are due to calibration and atmospheric effects and affect only the continuum shape.)

\section{Results}

\subsection{X-ray lightcurves}

The EPIC lightcurve for the first observation is best fit by a linear increase corresponding to a rise of $\sim 4 \%$ during the observation; the f-test yields a probability of $99.7 \%(3 \sigma)$ for the improvement in the fit compared to a constant flux value. The mean $0.2-10 \mathrm{keV}$ flux for this observation was $2.80 \pm 0.01 \times$ $10^{-12} \mathrm{erg} \mathrm{cm}^{-2} \mathrm{~s}^{-1}$. No significant variability is detected during the second observation, where the flux was $3.34 \pm 0.02 \times$ $10^{-12} \mathrm{erg} \mathrm{cm}^{-2} \mathrm{~s}^{-1}, 19 \%$ higher than the first. The spectrum hardened between the observations (from $\Gamma=2.58 \pm 0.02$ to $\Gamma=2.41 \pm 0.02$ ), consistent with previous observations of blazars, where an increase in the synchrotron flux frequently coincides with an increase in the synchrotron peak frequency (e.g. Pian et al. 1998; Wehrle et al. 1998).

\subsection{X-ray spectra}

The spectra from each epoch were found to be consistent, allowing for variability in the normalisation and slope of the power-laws. Both observations have therefore been fit simultaneously, allowing only the power-law component of the model to vary independently. All X-ray spectral models included neutral absorption fixed at the Galactic level $\left(6.28 \times 10^{20} \mathrm{~cm}^{-2}\right)$. It is clear however that there is absorption above the Galactic level in the X-ray spectra of this source (Fig. 2, see also Stocke et al. 1995; Watson et al. 1999); fitting a broken power-law to model the downturn at the soft end of the spectrum instead of absorption results in a very flat low energy slope $(\Gamma=0.75)$ and a fit considerably worse than that obtained with neutral absorption at $z=0.351$.

In each observation, fitting a power-law with neutral absorption at $z=0.351$ to the combined data gives an unacceptable fit statistic $\left(\chi^{2}=1697\right.$ for 1476 degrees of freedom, null hypothesis probability $\left.\left(N_{\mathrm{H}}\right)=5 \times 10^{-5}\right)$. The data deviate significantly from the model at low energies, implying that the absorption is different from that modelled or that the absorber is correct but that there is a different continuum below $\sim 1 \mathrm{keV}$. Such a difference in continuum at soft energies is often observed in Seyfert galaxies as a "soft excess", empirically modelled with one or more black-body components (e.g. Page et al. 2002). Adding a black-body component to the power-law with absorption at $z=0.351$ improved the fit significantly $\left(\chi^{2} /\right.$ d.o.f. $\left.=1584 / 1474, N_{\mathrm{H}}=0.02\right)$. The best-fit black-body temperature was $0.119_{-0.006}^{+0.008} \mathrm{keV}$ with luminosity $\sim 8 \times 10^{43} \mathrm{erg} \mathrm{cm}^{-2} \mathrm{~s}^{-1}$ at $z=0.351$. This model fits the data as well as models with variable redshift or variable abundances 
Table 1. Best-fit parameters and $68 \%$ confidence limits for various fits to the combined EPIC data. The basic model used was a power-law with neutral absorption fixed at the Galactic level $\left(6.28 \times 10^{20} \mathrm{~cm}^{-2}\right)$. Added to this was either i) a neutral, solar abundance absorber at $z=0.351$, ii) a neutral, solar abundance absorber at variable redshift, iii) an ionised, solar abundance absorber at variable redshift or iv) a neutral variable abundance absorber at $z=0.351$. Columns $1-6$ are the fitted model, absorbing column density, redshift, abundance and ionisation parameter, and the $\chi^{2}$ statistic over the number of degrees of freedom for the fit.

\begin{tabular}{|c|c|c|c|c|c|}
\hline Model & $\begin{array}{l}N_{\mathrm{H}} \\
\left(10^{20} \mathrm{~cm}^{-2}\right)\end{array}$ & $z$ & $Z$ & $\xi$ & $\frac{\chi^{2}}{\text { d.o.f. }}$ \\
\hline \multirow[t]{2}{*}{ i) } & 14.5 & 0.351 & $1.0^{f}$ & $0.0^{f}$ & 1696.9 \\
\hline & (14.0-14.9) & - & - & - & 1476 \\
\hline \multirow[t]{2}{*}{ ii) } & 83 & 2.1 & $1.0^{f}$ & $0.0^{f}$ & 1587.7 \\
\hline & $(70-97)$ & $(1.90-2.30)$ & - & - & 1475 \\
\hline \multirow[t]{2}{*}{ iii) } & 89 & 2.1 & $1.0^{f}$ & 0.003 & 1586.1 \\
\hline & $(67-74)$ & $(1.9-2.2)$ & - & $(0-0.011)$ & 1474 \\
\hline \multirow[t]{2}{*}{ iv) } & 92 & $0.351^{f}$ & 0.04 & $0.0^{f}$ & $\underline{1589.4}$ \\
\hline & $(81-103)$ & - & $(0.03-0.07)$ & - & 1475 \\
\hline
\end{tabular}

${ }^{f}$ Fixed.

(see Table 1), however the need to invoke an emission component in order to explain an essentially smooth absorbed continuum and the fact that a soft excess has never been reported in a BL Lac render it a less favoured alternative.

In order to test the nature of the absorber it was assumed that the spectrum was entirely synchrotron dominated. The redshift, ionisation state and metallicity of the absorber were tested in four models: i) a neutral, solar abundance absorber at $z=0.351$, ii) a neutral, solar abundance absorber at variable redshift, iii) an ionised, solar abundance absorber at variable redshift or iv) a neutral, variable abundance absorber at $z=0.351$. The results of these four fits to both datasets are presented in Table 1.

Allowing the redshift to vary (beyond $z=0.351$ ) significantly improved the fit $\left(\chi^{2} /\right.$ d.o.f. $=1587.7 / 1475, N_{\mathrm{H}}=0.02$, f-test probability $=4 \times 10^{-23}$ ), giving a best-fit redshift of $z=2.1 \pm 0.2$, though there is another comparable minimum in the $\chi^{2}$ space near $z=1.35$ (Fig. 3). The contraint on the redshift arises principally from the non-detection of a strong neutral oxygen absorption edge, placing the redshift above one. In the case where the absorber is ionised, there is no significant improvement in the fit over the neutral case $\left(\chi^{2} /\right.$ d.o.f. $=$ $1586.1 / 1474, N_{\mathrm{H}}=0.02$, f-test probability compared to the neutral absorber $=0.78$ ), and the ionisation parameter is not well constrained (Table 1). However, the lack of detection of strong O VII or O VIII edges also places the redshift well beyond $z=0.351$ for this model.

Since it is the non-detection of these edges that constrains the redshift of the absorber in the latter cases, it is not surprising that a low abundance absorber with a redshift fixed

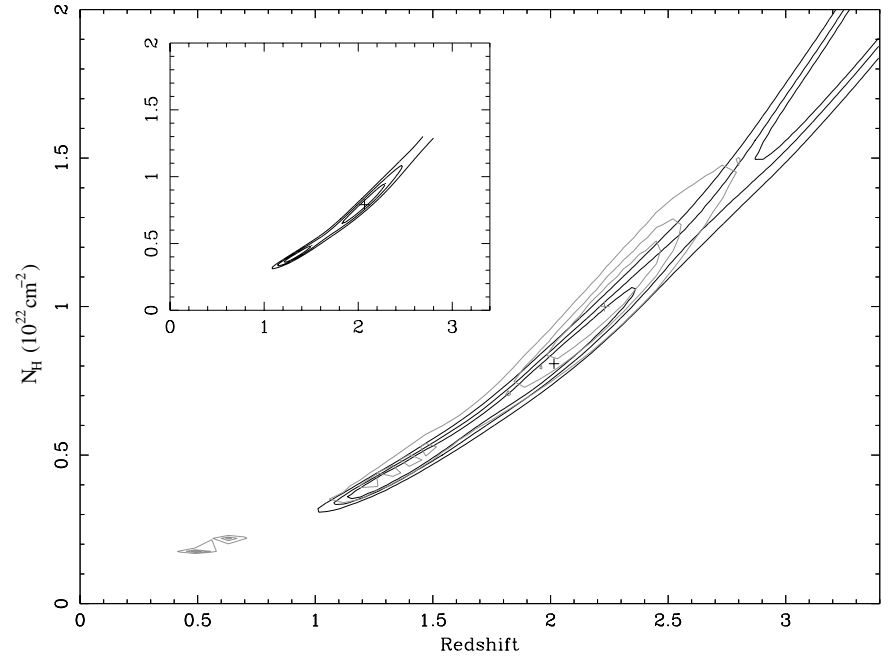

Fig. 3. Comparison of MOS-pn differences in the fit parameters, redshift and gas absorbing column density. Confidence contours are $1 \sigma$ (solid line), $2 \sigma$ (dashes) and $3 \sigma$ (dots) for fits to the EPIC-pn (black) and combined EPIC-MOS (grey) spectra from both observations. These parameters are clearly not significantly affected by MOSpn calibration differences. Inset. Confidence contours for the simultaneous fit of data from all EPIC cameras.

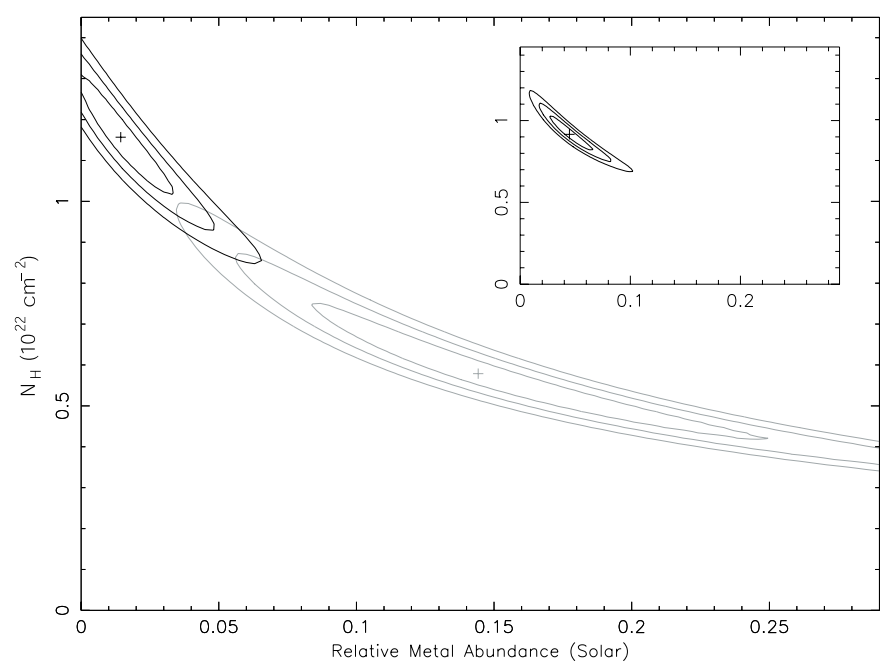

Fig. 4. Confidence contours $(1,2$ and $3 \sigma)$ for the best-fit metal abundances (relative to the Solar values) and gas absorbing column density to the EPIC spectra. EPIC-pn data are plotted in black, MOS in grey. The contours overlap only at greater than the $2 \sigma$ level. However, for both datasets the metal abundance is certainly less than half the solar value. Inset. Confidence contours for the simultaneous fit of data from all EPIC cameras.

at $z=0.351$ is statistically as good a fit as the higherredshift solar abundance absorbers $\left(\chi^{2} /\right.$ d.o.f. $=1589.4 / 1475$, $\left.N_{\mathrm{H}}=0.02\right)$. Allowing the redshift to vary in this case did not significantly improve the fit $\left(\chi^{2} /\right.$ d.o.f. $=1588.9 / 1474, \mathrm{f}$ test probability of 0.50 , compared to the model with redshift fixed at $z=0.351)$. There is some difference in the best-fit metal abundances determined from the MOS and pn datasets (see Fig. 4) due to calibration differences between the instruments; in particular, small systematic residuals may be fit as 
absorption edges in the MOS spectra. In any case, the abundance of the absorber must certainly be below half the solar value and is probably much lower (at the redshift inferred from the optical spectrum, $z=0.351$ ).

\section{Discussion and conclusions}

Observations with $A S C A$ indicated the peak of the synchrotron component in MS 0205.7+3509 to be between the UV and soft X-rays at the time of those observations (Watson et al. 1999), consistent with results from the first XMM-Newton observation. The harder spectrum and higher flux during the second observation imply a shift in the synchrotron peak frequency to higher energies.

The featureless nature of the absorbed spectrum implies either that the absorber is metal poor or that the absorbing material is at a redshift much higher than the $z=0.351 \mathrm{Mg}$ II absorption system detected in the optical spectrum. In either case the absorbing gas column density is similarly high $\left(\sim 10^{22} \mathrm{~cm}^{-2}\right)$, implying an optical extinction that is not observed $\left(A_{\mathrm{V}} \simeq 5\right.$ at $z=0$ where the gas to dust ratio is similar to the Galactic value, Bohlin et al. 1978), a fact that supports the low-redshift, low-metallicity model for the absorber.

It is possible that the absorber is associated with the BL Lac host galaxy. But the lack of gas and dust in elliptical galaxies (Knapp 1999), the typical hosts of BL Lacs, and the proximity of the companion galaxy (2.3", corresponding to an apparent linear distance of $\sim 11 \mathrm{kpc}$, Falomo et al. 1997) strongly suggest a link between the companion and the absorbing gas, as proposed by Falomo et al. (1997) and Watson et al. (1999). Furthermore, this is, as far as we are aware, the highest column density so far observed in the spectrum of a BL Lac object (after PKS 1413+135 which appears to have large absorption in its host galaxy, but the host in that case is an edge-on spiral, Perlman et al. 2002), implying that the absorption seen here is not associated with the BL Lac host. In this case the $z=0.351 \mathrm{Mg}$ II belongs to the X-ray absorbing gas which must be poor in dust and metals, implying that the BL Lac is illuminating a fairly pristine gas cloud that is associated with the galaxy close to the line of sight at redshift $z=0.351$.

\section{References}

Abraham, R. G., Crawford, C. S., Merrifield, M. R., Hutchings, J. B., \& McHardy, I. M. 1993, ApJ, 415, 101

Bohlin, R. C., Savage, B. D., \& Drake, J. F. 1978, ApJ, 224, 132

Burbidge, E. M., Beaver, E. A., Cohen, R. D., Junkkarinen, V. T., \& Lyons, R. W. 1996, AJ, 112, 2533

Falomo, R., Kotilainen, J., Pursimo, T., et al. 1997, A\&A, 321, 374

Gabuzda, D. C., Kollgaard, R. I., Roberts, D. H., \& Wardle, J. F. C. 1993, ApJ, 410, 39

Jansen, F., Lumb, D., Altieri, B., et al. 2001, A\&A, 365, L1

Knapp, G. R. 1999, in Star Formation in Early Type Galaxies, ASP Conf. Ser., 163, 119

Madejski, G. 1994, ApJ, 432, 554

Madejski, G., Takahashi, T., Tashiro, M., et al. 1996, ApJ, 459, 156

Morris, S. L., Stocke, J. T., Gioia, I. M., et al. 1991, ApJ, 380, 49

Nottale, L. 1986, A\&A, 157, 383

Ostriker, J. P., \& Vietri, M. 1990, Nature, 344, 45

Padovani, P. 1992, MNRAS, 257, 404

Page, K. L., Pounds, K. A., Reeves, J. N., \& O’Brien, P. T. 2002, MNRAS, 330, L1

Perlman, E. S., Stocke, J. T., Carilli, C. L., et al. 2002, AJ, 124, 2401

Pian, E., Falomo, R., Hartman, R. C., et al. 2002, A\&A, 392, 407

Pian, E., Vacanti, G., Tagliaferri, G., et al. 1998, ApJ, 492, L17

Rector, T. A., Stocke, J. T., Perlman, E. S., Morris, S. L., \& Gioia, I. M. 2000, AJ, 120, 1626

Romero, G. E., Cellone, S. A., \& Combi, J. A. 1999, A\&AS, 135, 477

Scarpa, R., \& Falomo, R. 1997, A\&A, 325, 109

Stocke, J. T., Wurtz, R. E., \& Perlman, E. S. 1995, ApJ, 454, 55

Strüder, L., Briel, U., Dennerl, K., et al. 2001, A\&A, 365, L18

Turner, M. J. L., Abbey, A., Arnaud, M., et al. 2001, A\&A, 365, L27

Urry, C. M., Falomo, R., Scarpa, R., et al. 1999, ApJ, 512, 88

Watson, D., Hanlon, L., McBreen, B., et al. 1999, A\&A, 345, 414

Watson, D., Smith, N., Hanlon, L., et al. 2000, A\&A, 364, 43

Wehrle, A. E., Pian, E., Urry, C. M., et al. 1998, ApJ, 497, 178 